\title{
Prevalence of primary open angle glaucoma in general ophthalmic practice in the United Kingdom
}

\author{
M Kroese, H Burton, S Vardy, T Rimmer, D McCarter
}

Br J Ophthalmol 2002;86:978-980

Aim: To estimate the predicted prevalence of primary open angle glaucoma (POAG) from the activity of a local ophthalmology department.

Method: Using clinic audit data, the local incidence and prevalence of POAG in the registered population of two primary care trusts were calculated.

Results: The local derived prevalence estimate for POAG was 978 per 100000 people aged $40-89$ years $(95 \% \mathrm{Cl}$ 753 to 1272) compared with the expected prevalence from a published model of 1230 people per 100000 people aged $40-89$ years.

Conclusion: The derived prevalence was not statistically significantly different from that predicted. Based on the published evidence that about half of the POAG cases are undetected, it would have been expected that local audit figures would have yielded figures about $50 \%$ lower than the epidemiological model. The main reason for this higher prevalence is thought to be differences in the diagnostic criteria used. This lack of consensus on the case definition for POAG is a deficit, which will hamper future needs assessment.

G laucoma is defined as "a progressive optic neuropathy involving characteristic structural damage to the optic nerve and characteristic visual field defects."1 Glaucoma can be classified into primary, secondary, and developmental glaucomas. This study concentrated on the incidence and prevalence of primary open angle glaucoma (POAG).

Glaucoma is the second most common cause of blindness as estimated from blindness certification. It accounts for $11.7 \%$ of all certifications for 1990-1 in England and Wales. ${ }^{2}$ There is an estimated prevalence of POAG for the age group 40-89 years in the white population of the United Kingdom of $1.2 \%{ }^{3}$ Consistent results have led to models for establishing the prevalence in white populations

The purpose of this study was to estimate the prevalence of POAG in general ophthalmic practice in the United Kingdom and compare it with the value obtained from a model based on epidemiological studies and thus to establish whether this model is a good predictor of service need presenting to the National Health Service.

\section{METHOD}

The ophthalmology department at Peterborough District Hospital provides a general ophthalmic service and does not have an ophthalmologist specialising in glaucoma care. Referrals are received from general practice, optometrists, and other hospital specialists.

New cases of POAG diagnosed in this department between l April 1999 and 31 March 2000 were identified through the department's ongoing audit programme. This programme includes the completion of a diagnostic code by a consultant ophthalmologist for every new patient. This process of diagnostic coding has been established for 3 years. The clinical diagnosis was made based on the evidence from the optic disc appearance, ocular pressure, and visual field testing as well as any changes that have occurred over time.

For each new POAG case, the age, sex, and primary care trust (PCT) of registration was recorded. Only new cases from North and South Peterborough PCTs were included in this study. Referrals from these two PCTs to other local ophthalmology departments were also checked for new POAG cases.

The denominator population was defined as those registered with the two PCTs in July 1999.

The observed incidence was calculated. Ninety five per cent confidence intervals (95\% CI) for the incidence and prevalence figures were calculated using the method outlined by Lilienfeld. ${ }^{4}$

Prevalence of POAG for the registered population in North and South Peterborough PCTs could not be established from the audit data and was therefore calculated from the incidence figures.

The relation between prevalence and incidence is represented by the equation:

Prevalence $=$ Cumulative incidence $\times$ Average duration of the disease

This equation is used to calculate the prevalence from the cumulative incidence.

There are three important assumptions in the calculation of the prevalence for POAG. The first is that the incidence is fixed or constant over time; secondly, that people with POAG have a normal life expectancy; and the third is that the population is a stable one. There is some evidence to support the second assumption. ${ }^{5}$ For the purposes of calculating the duration of illness for each case, the most recent life tables for England and Wales were used. ${ }^{6}$ The date of onset is assumed to be the same as the date of diagnosis. This is also an important assumption because POAG is asymptomatic until a late stage and therefore presentation for diagnosis will on average be after a long period of disease development. This assumption will therefore result in an underestimate in the duration of the disease and hence a lower prevalence estimate for the true population prevalence figure.

\section{RESULTS}

The number of new cases of POAG diagnosed in the registered populations of North and South Peterborough PCTs in the year l April 1999 until 31 March 2000 is shown by age band in Table 1 . The prevalence for POAG in the study group was 978 per 100000 people aged $40-89$ years (95\% CI 753 to 1272 ).

\section{DISCUSSION}

The results of this study were compared with the expected prevalence obtained from a recent epidemiological model developed by Tuck and Crick $^{3}$ which is based on data from eight major population surveys $\mathrm{s}^{7-14}$ and is applicable to white populations. The prevalence of POAG in the study population 
Table 1 New cases of POAG in North and South Peterborough PCTs 1 April 1999-31 March 2000

\begin{tabular}{llll}
\hline Age band (years) & $\begin{array}{l}\text { Total number of } \\
\text { cases }\end{array}$ & $\begin{array}{l}\text { Number of } \\
\text { registered } \\
\text { population }\end{array}$ & Incidence per 100 000/year observed $(95 \% \mathrm{Cl})$ \\
\hline$<40$ & 1 & 114089 & $28.68(7.80$ to 73.42$)$ \\
$40-44$ & 4 & 13945 & $0(0$ to 29.20$)$ \\
$45-49$ & 0 & 13332 & $7.60(0.19$ to 42.33$)$ \\
$50-54$ & 1 & 13155 & $60.70(22.28$ to 132.33$)$ \\
$55-59$ & 6 & 9884 & $33.57(6.92$ to 98.05$)$ \\
$60-64$ & 3 & 8934 & $122.24(58.68$ to 224.94$)$ \\
$65-69$ & 10 & 8180 & $136.29(62.43$ to 258.97$)$ \\
$70-74$ & 10 & 7337 & $226.86(123.87$ to 381.14$)$ \\
$75-79$ & 14 & 6171 & $393.31(169.52$ to 774.82$)$ \\
$80-84$ & 7 & 3330 & \\
$85-89$ & 8 & 2034 & Observed incidence for age group 40-89 years, \\
$>90$ & 2 & 972 & $73(56.21$ to 94.90$)$ \\
Total & 66 & 201363 & \\
& & &
\end{tabular}

was 978 per 100000 people aged $40-89$ years (95\% CI 753 to 1272) compared to the expected prevalence from the Tuck/Crick model of 1230 per 100000 people aged 40-89 years. The difference in prevalence estimates was not statistically significant.

These findings were surprising given that we were comparing an audit of patients known to services with a population based survey. Previous surveys which were population or community based rather than based on people using services have found that about $50 \%$ of the POAG cases were unknown to medical services. ${ }^{7-10} 1516$

We considered the possibility that these finding were due to error either in the denominator population or in the completion of coding forms. These sources were not thought to be significant and, if they existed, would be more likely to lead to an underestimate of prevalence rather than an overestimate.

The results obtained could be an artefact because of a recent change in the practice of optometrists or ophthalmologists during the year resulting in a sudden and possibly temporary increase in POAG cases referred and diagnosed. However, clinicians confirmed that there was no recent change in ophthalmic practice nor a dramatic increase in referrals from optometrists in the time period of the study.

After discounting these main sources of error, we were left with three main possibilities to account for the high level of POAG. Firstly, that our clinic was detecting a higher proportion of cases in the community than usual. In other words, there was a larger proportion of the clinical iceberg of total cases being recognised. This appears to be unlikely since local population screening practice by optometrists is broadly similar to elsewhere in the United Kingdom.

Secondly, the results obtained may be explained by the use of different diagnostic criteria by the clinic from those used in the surveys which provided the results for the Tuck/Crick model. It was only the data on "definite" cases of POAG that were used in the Tuck/Crick model. The authors themselves admit that if "probable" cases were included the prevalence for people aged $40-89$ years would be higher at $1.5-2.0 \% .^{3}$ It is also likely that in the absence of agreed guidelines on the diagnosis of POAG in the Peterborough clinic, a number of "suspect" cases have been included in the observed data. This difference in diagnostic criteria is the most likely explanation.

Finally, the results could reflect a true increased prevalence of "definite" POAG among the population of the two PCTs. This could only be investigated by an ophthalmic survey of the two PCT populations. However, there are no features of the combined population of the two PCTs to suggest a reason for an increased incidence and prevalence of POAG. The population defined has a normal cross section of socioeconomic status, contains a mix of urban and rural communities with an age distribution similar to the national population, and is $94 \%$ white.

\section{CONCLUSIONS}

We found an estimate of POAG prevalence known to a local district hospital which may be of use to those planning services. This estimate is higher than that which would be predicted using a theoretical epidemiological model based on measured population prevalences, since it is usually held that only half the cases of POAG are known to services. What proportion of the cases in the NHS clinic are "true" POAG depends on what definition is used. However, even if, as is likely, a proportion of these cases are false positives and do not have the condition, their treatment by the NHS ophthalmology outpatient service is an important finding and must be taken into account by commissioners of services and ophthalmologists when planning services. This study provides evidence that the actual number of cases being treated as POAG in the NHS is much higher than expected.

In the long run, for the better assessment of need and to facilitate local audit, it would be advantageous to reach a consensus on a workable clinical definition of POAG in the United Kingdom. This definition can then be used to develop a new model for estimating the prevalence of POAG.

\section{Authors' affiliations}

M Kroese, Eastern Region Public Health Observatory, Institute of Public Health, Box 113, University Forvie Site, Robinson Way, Cambridge, CB2 2SR, UK

H Burton, Public Health Genetics Unit, Strangeways Reasearch Laboratory, Worts Causeway, Cambridge, CB1 8RN, UK

S Vardy, T Rimmer, D McCarter, Department of Ophthalmology, Peterborough District Hospital, Thorpe Road, Peterborough PE3 6DA, UK

Correspondence to: Mark Kroese

Competing interests: none declared.

Accepted for publication 10 April 2002

\section{REFERENCES}

1 Gupta N, Weinreb RN. New definitions of glaucoma. Curr Opin Ophthalmol 1997;8:38-41.

2 Evans J. Causes of blindness and partial sight in England and Wales 1990-1991. London: HMSO, 1995;1-29

3 Tuck MW, Crick RP. The age distribution of primary open angle glaucoma [published erratum appears in Ophthalmic Epidemiol 1999;6:84]. Ophthalmic Epidemiol 1998;5:173-83.

4 Lilienfield AM, Lilienfield DE. Foundations of epidemiology. Oxford: Oxford University Press, 1980:336-8. 
5 Klein R, Klein BE, Moss SE. Age-related eye disease and survival. The Beaver Dam Eye Study. Arch Ophthalmol 1995;113:333-9.

6 Office for National Statistics. English life tables no 15 1990-1992. London: Stationery Office, 1997:1-20.

7 Dielemans I, Vingerling JR, Wolfs RC, et al. The prevalence of primary open-angle glaucoma in a population-based study in the Netherlands.

The Rotterdam Study. Ophthalmology 1994;101:1851-5.

8 Giuffre G, Giammanco R, Dardanoni G, et al. Prevalence of glaucoma and distribution of intraocular pressure in a population. The Casteldaccia Eye Study. Acta Ophthalmol Scand 1995;73:222-5.

9 Coffey M, Reidy A, Wormald R, et al. Prevalence of glaucoma in the west of Ireland. Br J Ophthalmol 1993;77:17-21.

10 Mitchell P, Smith W, Attebo K, et al. Prevalence of open-angle glaucoma in Australia. The Blue Mountains Eye Study. Ophthalmology

1996;103:1661-9.
11 Leske MC, Ederer F, Podgor M. Estimating incidence from age-specific prevalence in glaucoma. Am J Epidemiol 1981;113:606-13.

12 Hollows FC, Graham PA. Intra-ocular pressure, glaucoma, and glaucoma suspects in a defined population. $\mathrm{Br} J$ Ophthalmol 1966:50:570-86.

13 Tielsch JM, Sommer A, Katz J, et al. Racial variations in the prevalence of primary open-angle glaucoma. The Baltimore Eye Survey. JAMA 1991;266:369-74.

14 Klein BE, Klein R, Sponsel WE, et al. Prevalence of glaucoma. The Beaver Dam Eye Study. Ophthalmology 1992:99:1499-504.

15 Wensor MD, McCarty CA, Stanislavsky YL, et al. The prevalence of glaucoma in the Melbourne Visual Impairment Project. Ophthalmology 1998;105:733-9.

16 Crick RP. Epidemiology and screening of open-angle glaucoma. Curr Opin Ophthalmol 1994:5:3-9.

\section{Adding insult to injury?}

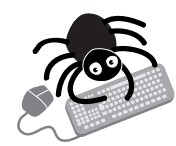

Please visit the British Journal of

Ophthalmology website [www. bjophthalmol.com for link to this full article.
Isually impaired people are continuing to suffer accidental injuries related to their impairment because epidemiological research of the subject lacks rigour and intervention studies are almost non-existent.

A recent review of published data calls for comprehensive studies to identify the causes of injury and evaluate preventive measures, and it recommends minimum standards for future studies. These include formal ophthalmological assessment of visual acuity and visual fields, measurement of injuries as outcomes, and adjustment for confounders.

Of 31 studies that conformed to the review's inclusion criteria, about two thirds concerned falls, eight traffic related injuries, and three occupational injuries. Other types of accidents were not covered. Most studies were inconclusive-through having insufficient power or no adjustment for confounders, or both.

Those few studies with meaningful results dealt with falls in older people. The risk of falls in visually impaired people aged $>75$ years was 1.7 times that of sighted people. Multiple falls were 1.2-2.1 times more likely, depending on the type of impairment. The risk of hip fracture was 1.3-1.9 times higher for people with lowered visual acuity.

Research studies published during 1980-2000 were identified by MeSH and free text searches of 10 bibliographic databases. Only those which satisfied two or more of the following criteria were included in the review: formal ophthalmic assessment; adjustment for confounders; large sample size, with numbers of visually impaired subjects; and clear definitions and outcomes.

Visually impaired people are at increased risk of injury and therefore an important consideration in any measures to reduce injuries in general.

A Injury Prevention 2002;8:155-160. 